\title{
B7-H1-mediated immunosuppressive properties in human mesenchymal stem cells are mediated by STAT-1 and not PI3K/Akt signaling
}

\author{
IN KEUN JANG ${ }^{1,2 *}$, HYUN JOO JUNG ${ }^{1 *}$, O KYU NOH $^{3,4}$, DOO-HOON LEE ${ }^{2}$, \\ KWANG CHUL LEE ${ }^{5}$ and JUN EUN PARK ${ }^{1}$
}

\author{
${ }^{1}$ Department of Pediatrics, Ajou University School of Medicine, Suwon 16499; ${ }^{2}$ Biomedical Research Institute, \\ Lifeliver Co., Ltd., Yongin 16866; Departments of ${ }^{3}$ Radiation Oncology and ${ }^{4}$ Biomedical Informatics, \\ Ajou University School of Medicine, Suwon 16499; ${ }^{5}$ Department of Pediatrics, \\ College of Medicine, Korea University, Seoul 02841, Republic of Korea
}

Received August 20,2017; Accepted May 1, 2018

DOI: $10.3892 / \mathrm{mmr} .2018 .9102$

\begin{abstract}
Mesenchymal stem cells (MSCs), derived from either bone marrow (BM) or Wharton's jelly (WJ), inhibit the proliferation of activated $\mathrm{T}$ cells, and interferon (IFN) $-\gamma$ serves an important role in this process. This process is B7-homolog (H)1-dependent during cell contact inhibition. However, the signaling pathway involved in B7-H1 expression in MSCs remains largely undefined. The present study demonstrated activation of B7-H1 by engaging signal transducer and activator of transcription (STAT)-1 signaling in MSCs. Human BM- and WJ-MSCs were isolated and cultured. The immunosuppressive effect of BM- and WJ-MSCs on phytohemagglutinin (PHA)-induced $\mathrm{T}$ cell proliferation was compared using direct and indirect co-culture systems. B7-H1 expression on BM- and WJ-MSCs was detected by flow cytometry. Small interfering (si)RNA was used to knock down the expression of STAT-1. The inhibitory effect of MSCs on T lymphocytes was observed using PHA-induced $\mathrm{T}$ cell proliferation assays. IFN- $\gamma$-induced $\mathrm{B} 7-\mathrm{H} 1$ expression on human BM- and WJ-MSCs increased in a time-dependent manner. Furthermore, the inhibitory effect of MSCs on T cell proliferation was be restored when an anti-B7-H1 monoclonal antibody was used. When STAT-1 signaling was inhibited by siRNA, B7-H1 expression on IFN- $\gamma$-treated MSCs decreased and $\mathrm{T}$ cell proliferation was restored; however, the expression
\end{abstract}

Correspondence to: Dr Jun Eun Park, Department of Pediatrics, Ajou University School of Medicine, 164 Worldcup-ro, Yeongtong-gu, Suwon 16499, Republic of Korea

E-mail: pedpje@ajou.ac.kr

*Contributed equally

Key words: B7-homolog 1, signal transducer and activator of transcription 1, mesenchymal stem cells, interferon- $\gamma$, phosphatidylinositol-3-kinase/RAC- $\alpha$ serine/threonine-protein kinase of B7-H1 did not alter upon treatment with a phosphatidylinositol-3-kinase (PI3K) inhibitor (LY294002). These results demonstrated that the IFN- $\gamma$-induced immunosuppressive properties of B7-H1 in human BM- and WJ-MSCs were mediated by STAT-1 signaling, and not by PI3K/RAC- $\alpha$ serine/threonine-protein kinase signaling. Understanding the intracellular mechanisms underlying IFN- $\gamma$-induced expression of B7-H1 in MSCs may ultimately lead to an improved understanding of MSCs and provide insight into their use as cell therapy agents.

\section{Introduction}

Mesenchymal stem cells (MSCs) were first isolated from bone marrow (BM) by Friedenstein et al (1) and have since been isolated from various tissues including BM, umbilical cord or Wharton's Jelly (WJ), umbilical cord blood, placenta, and adipose tissue, and are also present in the connective tissue of the majority of organs (2). The minimal criteria for defining MSCs include attachment in standard systems, surface expression of cluster of differentiation (CD)73, CD90, and CD105, lack of expression of CD34, CD45, CD11b or CD14, CD19 or CD79a and human leukocyte antigen (HLA)-DR, and the ability to differentiate into osteoblasts, chondroblasts, and adipocytes $(3,4)$.

Previously, several experiments have been performed to exploit the immunoregulatory and cytotoxic properties of MSCs as therapeutics $(4,5)$. There are several properties that make these cells appropriate as cell therapy agents. First, MSCs express very low levels of HLA class I molecules, and HLA class II and co-stimulatory molecules (CD80, CD86, or CD40) are rarely expressed $(5,6)$. Although an immune barrier has been noted with the use of MSCs in clinical trials, these cells are known to be fully available for allogeneic and autologous stem cell transplants due to this unique immunologic property (4). Second, MSCs may easily be obtained from the WJ (WJ-MSCs) of the human umbilical cord, which is discarded along with the placenta following childbirth. These cells may be isolated from almost $100 \%$ of cord samples at a markedly 
greater frequency compared with other tissues including BM, adipose tissue, and cord blood.

Despite the potential of MSCs to be used as therapeutic agents, their immunoregulatory function tends to depend on different culturing conditions and the cytokine environment; thus, further research is required (4). Regarding immunosuppressive effects, although the associated mechanisms of soluble factors including indoleamine 2,3-dioxygenase (IDO) are known, intracellular mechanisms based on direct contact are relatively unknown. Interferon (IFN)- $\gamma$-induced IDO production has been demonstrated to be involved in the downstream activation of IFN- $\gamma$ receptors including signal transducer and activator of transcription (STAT) proteins and the phosphatidylinositol 3-kinase (PI3K) pathway (7).

B7-homolog $(\mathrm{H}) 1$, also referred to as CD274 or programmed death ligand 1 (PD-L1), is present in various tissues and acts as an inhibitory co-stimulatory surface molecule during immune responses, and functions through cell-to-cell contact $(8,9)$. Although B7-H1 is constitutively expressed in naïve MSCs, IFN- $\gamma$ induces significant upregulation of B7-H1 in these cells (10). Based on T cell proliferation assays, when B7-H1 is blocked, MSC-mediated suppression of T cells is inhibited and $\mathrm{T}$ cell proliferation in the presence of anti-B7-H1-treated MSCs is restored to $55-70 \%$ of control levels (11). To a certain extent, it is known that signaling pathways induce immunosuppression by regulating proliferation and differentiation via the release of soluble factors including IDO from MSCs (12). However, the signaling pathway involved in B7-H1 expression in MSCs remains to be fully elucidated (13). The present study investigated the effect of IFN- $\gamma$ receptor-binding on Janus kinase (Jak)-transcriptional signal transduction and the transcriptional activation of STAT and PI3K/RAC- $\alpha$ serine/threonine-protein kinase (Akt) pathways, to understand the signal transduction mechanisms and transcriptional regulation of $\mathrm{B} 7-\mathrm{H} 1$ in MSCs.

\section{Materials and methods}

Isolation and culture of human BM- and WJ-derived MSCs. The Institutional Review Board of Ajou University Hospital (Suwon, Korea) approved the present study (AJIRB-GN3-07-208), and all samples were obtained with informed consent. The BM-MSC donor was a healthy man in his twenties with no infectious disease and no family history of hereditary disease. The UC-MSC donor was a woman in her thirties who underwent a caesarian section.

BM-MSCs were isolated and cultured as described previously (14). Briefly, mononuclear cells were isolated from normal BM aspirates using Ficoll-Hypaque density gradient centrifugation at $350 \mathrm{x}$ g for $30 \mathrm{~min}$ at $20^{\circ} \mathrm{C}$ (Histopaque-1077; Sigma-Aldrich; Merck KGaA, Darmstadt, Germany). Cells were seeded at a density of $3 \times 10^{5}$ cells $/ \mathrm{cm}^{2}$ in low glucose Dulbecco's modified Eagle's medium (LG-DMEM; Gibco; Thermo Fisher Scientific, Inc., Waltham, MA, USA) supplemented with $10 \%$ fetal bovine serum (FBS; Invitrogen-Gibco; Thermo Fisher Scientific, Inc.) and $100 \mathrm{U} / \mathrm{ml}$ penicillin/streptomycin (Invitrogen-Gibco; Thermo Fisher Scientific, Inc.). Cells were incubated in a humidified atmosphere at $37^{\circ} \mathrm{C}$ with 5\% $\mathrm{CO}_{2}$, and the medium was replaced every 3-4 days until the adherent fibroblast-like cells reached confluence.
Adherent cells were then resuspended in $0.05 \%$ trypsin-EDTA (Invitrogen-Gibco; Thermo Fisher Scientific, Inc.) and reseeded at a concentration of $2 \times 10^{3}$ cells $/ \mathrm{cm}^{2}$. WJ-MSCs were isolated using an explantation culture method (15). Umbilical cords were washed with Dulbecco's phosphate-buffered saline to remove blood components and cut into small pieces $(0.5-1 \mathrm{~cm})$. Vessels were removed to avoid endothelial cell contamination. The WJ components of the cord were cut into $0.5-1 \mathrm{~cm}^{3}$ pieces and placed directly into culture wells for expansion in LG-DMEM supplemented with $10 \% \mathrm{FBS}$ and $100 \mathrm{U} / \mathrm{ml}$ penicillin/streptomycin. When colonies of cells appeared and reached $70 \%$ confluence, cells were resuspended in $0.05 \%$ trypsin-EDTA and reseeded at $2 \times 10^{3}$ cells $/ \mathrm{cm}^{2}$. In certain experiments, MSCs were treated with 1,000 U/ml IFN- $\gamma$ (LG Life Sciences Ltd., Seoul, Korea) and/or $10 \mu \mathrm{M}$ LY294002 (Cell Signaling Technology, Inc., Danvers, MA).

RNA interference. MSCs were plated $24 \mathrm{~h}$ prior to small interfering (si)RNA transfection such that cells reached $50 \%$ confluence on the day of transfection. Cells $\left(1 \times 10^{4}\right.$ cells $\left./ \mathrm{cm}^{2}\right)$ were incubated with siRNA-Lipofectamine 2000 (Invitrogen; Thermo Fisher Scientific, Inc.) complexes for $12 \mathrm{~h}$ at $37^{\circ} \mathrm{C}$. The medium was then replaced, and the transfected cells were incubated for an additional $12 \mathrm{~h}$ until the target gene was effectively downregulated. siRNAs targeting STAT-1 (sc-61879) and scrambled siRNA (sc-37007) were purchased from Santa Cruz Biotechnology, Inc. (Dallas, TX, USA).

Assessment of $T$ cell proliferation by 5-bromo-2'-deoxyuridine (BrdU) incorporation. MSCs were seeded at $3.75 \times 10^{4}$ cells $/ \mathrm{cm}^{2}$ in high-glucose DMEM supplemented with $10 \% \mathrm{FBS}$ and $100 \mathrm{U} / \mathrm{ml}$ penicillin/streptomycin. Following $24 \mathrm{~h}, 10 \mu \mathrm{g} / \mathrm{ml}$ mitomycin C (MMC; Sigma-Aldrich; Merck $\mathrm{KGaA}$ ) was added to inhibit MSC proliferation, and cells were incubated for $2 \mathrm{~h}$ at $37^{\circ} \mathrm{C}$ followed by 5 extensive washes with medium. Peripheral blood mononuclear cells (PBMCs) from a healthy volunteer were isolated by density gradient centrifugation $\left(350 \mathrm{xg}\right.$ for $30 \mathrm{~min}$ at $\left.20^{\circ} \mathrm{C}\right)$. PBMCs $\left(3 \times 10^{5} \mathrm{PBMCs} / \mathrm{cm}^{2}\right)$ were added and stimulated with $1 \mu \mathrm{g} / \mathrm{ml}$ phytohemagglutinin (PHA; Sigma-Aldrich; Merck KGaA). For Transwell assays, PBMCs were added to the upper wells of the Transwell plate (pore size $0.4 \mu \mathrm{m}$ ). PHA-activated PBMCs were cultured from the upper chamber in the presence or absence of MSCs, which were in the lower chamber. Cultures were plated in triplicate and incubated for 4 days prior to the addition of BrdU. Following $18 \mathrm{~h}$, proliferation was assessed using the BrdU Assay kit (Roche Applied Science, Penzberg, Germany) according to the manufacturer's protocol. In certain experiments, MSCs were treated with anti-B7-H1 (eBioscience; Thermo Fisher Scientific, Inc.).

Flow cytometric analysis of surface antigen expression. Antibodies against B7-H1 phycoerythrin (PE)-conjugated (anti-human B7-H1 monoclonal antibody; catalog no. 5983; $0.5 \mu \mathrm{g} / 0.2 \mathrm{ml}$ ) were purchased from eBioscience (Thermo Fisher Scientific, Inc.). A total of $5 \times 10^{5}$ cells were resuspended in $0.2 \mathrm{ml}$ PBS and incubated with the B7-H1-PE antibodies for $20 \mathrm{~min}$ at room temperature. The fluorescence intensity of cells was evaluated by flow cytometry using an 
FC 500 flow cytometer (Beckman Coulter, Brea, CA, USA), and the data were analyzed with CXP analysis version 2.0 (Beckman Coulter).

Reverse transcription-polymerase chain reaction analysis $(R T-P C R)$. Total RNA from MSCs was isolated using a QIAGEN RNeasy Mini Kit (Qiagen GmbH, Hilden, Germany), and was used to perform semi-quantitative RT-PCR assays using a commercial kit (PrimeScript 1st strand cDNA synthesis kit; Takara Bio, Inc., Otsu, Japan). PCR was performed using DNA polymerase Taq DAN polymerase kit (Koma Biotech, Seoul, Korea) and the primer sequences were B7-H1, forward: 5'-GACCTGAAGGTTCAG CATAG-3', reverse: 5'-GTATCTTGGATGCCACATTT-3', GAPDH, forward: 5'-ATCACCATCTTCCAGGAGCG-3', reverse: 5'-CCTGCTTCACCACCTTCTTG-3'. The following conditions were employed: 30 cycles consisting of a $5 \mathrm{~min}$ presoak at $94^{\circ} \mathrm{C}$, denaturation for $30 \mathrm{sec}$ at $94^{\circ} \mathrm{C}$, annealing for $30 \mathrm{sec}$ at $58^{\circ} \mathrm{C}$, and extension for $1 \mathrm{~min}$ at $72^{\circ} \mathrm{C}$, with an additional $10 \mathrm{~min}$ incubation at $72^{\circ} \mathrm{C}$ after completion of the cycle. Amplified cDNA fragments were electrophoresed on $2 \%$ agarose gel, stained with ethidium bromide $(0.5 \mu \mathrm{g} / \mathrm{ml}$; Biosesang, Seoul, Korea), and images captured under an ultraviolet light transilluminator (Bio-Rad Laboratories, Inc., Hercules, CA, USA).

Western blotting. MSCs were washed with cold PBS and lysed in $300 \mu \mathrm{l}$ of cold radioimmunoprecipitation buffer [50 mM tris- $\mathrm{HCl}$, pH 7.5, containing $1 \%$ Triton X-100, $150 \mathrm{mM} \mathrm{NaCl}$, $0.1 \%$ sodium dodecyl sulfate (SDS), $1 \%$ sodium deoxycholate, and a protease inhibitor cocktail (Thermo Fisher Scientific, Inc.)]. Cell lysates were centrifuged at 3,000 x g for $15 \mathrm{~min}$ at $4^{\circ} \mathrm{C}$. The supernatant was collected, and protein concentrations were analyzed using a bicinchoninic acid protein assay kit (Thermo Fisher Scientific, Inc.). For electrophoresis, proteins $(50 \mu \mathrm{g})$ were dissolved in sample buffer $(60 \mathrm{mM}$ tris- $\mathrm{HCl}, \mathrm{pH} 6.8$, containing $14.4 \mathrm{mM} \beta$-mercaptoethanol, $25 \%$ glycerol, $2 \%$ SDS, and $0.1 \%$ bromophenol blue), boiled for 5 min, and separated on a 10\% SDS reducing gel. Separated proteins were transferred onto polyvinylidene difluoride membranes (GE Healthcare Life Sciences, Little Chalfont, UK) using a trans-blot system (Invitrogen-Gibco; Thermo Fisher Scientific, Inc.). Blots were blocked for $1 \mathrm{~h}$ at room temperature in tris-buffered saline (TBS; $10 \mathrm{mM}$ Tris- $\mathrm{HCl}$, $\mathrm{pH} 7.5$, plus $150 \mathrm{mM} \mathrm{NaCl}$ ) containing $5 \%$ nonfat dry milk (BD Biosciences, San Jose, CA, USA), washed 3 times with TBS, and incubated at $4{ }^{\circ} \mathrm{C}$ overnight with primary antibodies in TBST (TBS plus $0.01 \%$ Tween-20) containing 3\% nonfat dry milk. On the next day, blots were washed three times with TBST and incubated for $1 \mathrm{~h}$ with secondary antibodies in TBST containing $3 \%$ nonfat dry milk at room temperature. Following washing three times with TBST, proteins were visualized with an enhanced chemiluminescence detection system (GE Healthcare Life Sciences). Antibodies used in this study were anti-STAT-1 (rabbit anti-human polyclonal antibody; catalog no. 9172; 1:1,000), anti-phospho (p)-STAT-1 (rabbit anti-human monoclonal antibody; catalog no. 9018; 1:1,000), anti-pAkt1-ser473 (rabbit anti-human monoclonal antibody; catalog no. 9018; 1:1,000), and horseradish peroxidase-conjugated anti-rabbit IgG (goat anti-rabbit polyclonal antibody; catalog no. 7074; 1:1,000; all from Cell Signaling Technology, Inc.) together with anti-actin (rabbit anti-human polyclonal antibody; catalog no. SC-1615; 1:1,000; Santa Cruz Biotechnology, Inc.).

Statistical analysis. Data are expressed as the mean \pm standard deviation. Differences between experimental conditions were analyzed by Student's t-test or one-way analysis of variance with Duncan's test. All statistical analyses were performed with IBM SPSS statistics software, version 19.0 (IBM Corp., Armonk, NY, USA). $\mathrm{P}<0.05$ was considered to indicate a statistically significant difference.

\section{Results}

BM- and WJ-MSCs inhibit T cell proliferation via B7-H1. To verify the ability of BM- and WJ-MSCs to inhibit T cell proliferation by direct contact, Transwell assays were used. It was verified that the inhibitory effect was due to direct contact based on the different results obtained for mixed culture and separation culture (Fig. 1A). The expression of B7-H1, which inhibits $\mathrm{T}$ cell activation by direct contact was verified. Untreated MSCs were also compared with IFN- $\gamma$-treated groups based on our previous findings that IFN $-\gamma$ improves the immunosuppressive ability of MSCs (11). As a result, the expression of B7-H1 was markedly increased by IFN- $\gamma$ (Fig. 1B). T cell proliferation in anti-B7-H1 treated MSCs was significantly restored compared to no antibody treatment (Fig. 1C).

IFN- $\gamma$ activates B7-H1 expression in MSCs in a time- and dose-dependent manner. The expression of $\mathrm{B} 7-\mathrm{H} 1$ by MSCs was determined following treatment with IFN- $\gamma$ at various concentrations $(0,1,10,100$ and $1,000 \mathrm{U} / \mathrm{ml})$ for $24 \mathrm{~h}$. The expression of B7-H1 increased dose-dependently in BM and WJ MSCs (Fig. 2A). In addition, treatment with $1,000 \mathrm{U} / \mathrm{ml}$ resulted in alterations over time $(0,6,12,24,48$ and $72 \mathrm{~h})$. Expression increased rapidly following $12 \mathrm{~h}$ and highest levels were observed between at 48 h (Fig. 2B).

IFN- $\gamma$-induced activation of B7-HI in MSCs is mediated by STAT-1 signaling. Alterations in STAT-1 expression, one of the signaling pathways activated by IFN- $\gamma$, were next assessed. It was verified that phospho-STAT-1 was elevated by IFN- $\gamma$ treatment (Fig. 2C), and increased from $10 \mathrm{U} / \mathrm{ml}$, which was similar to the B7-H1 expression pattern observed in Fig. 2A. siRNA was used to downregulate STAT-1 expression and decreased expression was observed when cells were treated with STAT-1 siRNA alone or with IFN- $\gamma$ (Fig. 3A). It was demonstrated that the expression of B7-H1 was abruptly inhibited through STAT-1 siRNA treatment (Fig. 3B). In addition, T cell proliferation in STAT-1 siRNA treated MSCs was significantly restored compared to no siRNA treatment, similar to that observed with anti-B7-H1 treatment (Fig. 3C).

IFN- $\gamma$-induced activation of B7-HI in MSCs is not mediated by PI3K/Akt signaling. The alterations in PI3K/Akt induction, another signaling pathway activated by IFN $-\gamma$, were 

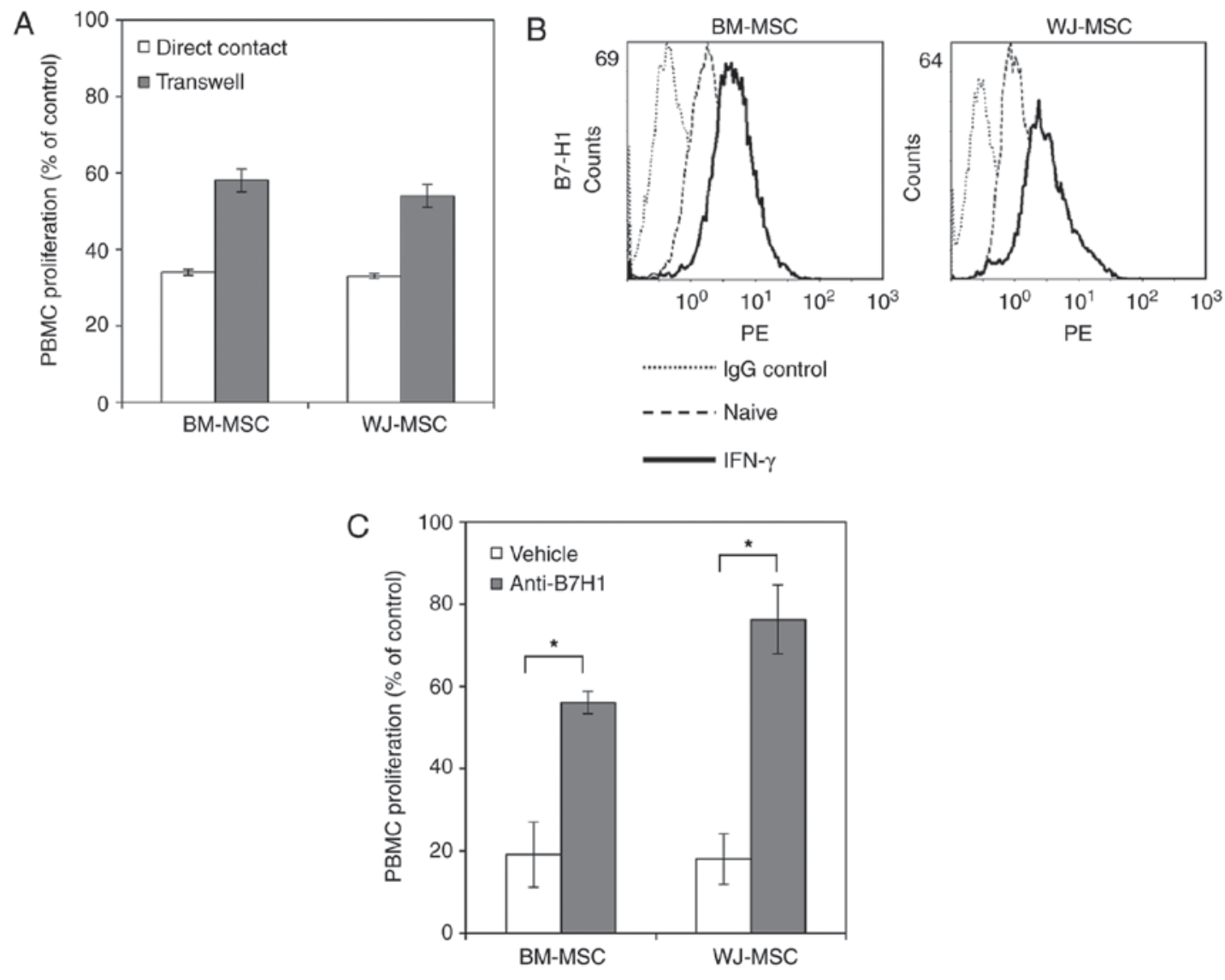

Figure 1. Immunosuppressive potential and immunophenotype of BM and WJ MSCs. (A) Phytohemagglutinin-induced proliferation of T cells from PBMCs, in the absence or presence of MSCs in direct contact (white bar) or indirect contact using a Transwell (gray bar) system was evaluated on day 3 as the percentage of BrdU+ cells. Error bars represent standard deviation. (B) BM- and WJ-MSCs were stimulated in the presence or absence of $1,000 \mathrm{U} / \mathrm{ml} \mathrm{IFN-} \gamma$ for $24 \mathrm{~h}$. B7-H1 expression was analyzed by flow cytometry (dotted line, IgG control; fine line, naïve MSCs; bold line, IFN- $\gamma$-treated MSCs). (C) Blocking B7-H1 in MSCs prevented MSC-mediated suppression of T cells based on proliferation assays. T cell proliferation in anti-B7-H1 treated MSCs was significantly restored compared to no antibody treatment ( ${ }^{*} \mathrm{P}<0.05$; error bars represent standard deviation). The proliferation of $\mathrm{T}$ cells stimulated with phytohemagglutinin-induced PBMCs alone was used as a control. Data measured were relative to this control. BrdU, 5-bromo-2'-deoxyuridine; IFN, interferon; PE, phycoerythrin-conjugated antibodies; bm, bone marrow; WJ, Wharton's jelly; MSCs, mesenchymal stem cells; PBMCs, peripheral blood mononuclear cells; B7-H1, B7 homolog 1; IgG, immunoglobin.
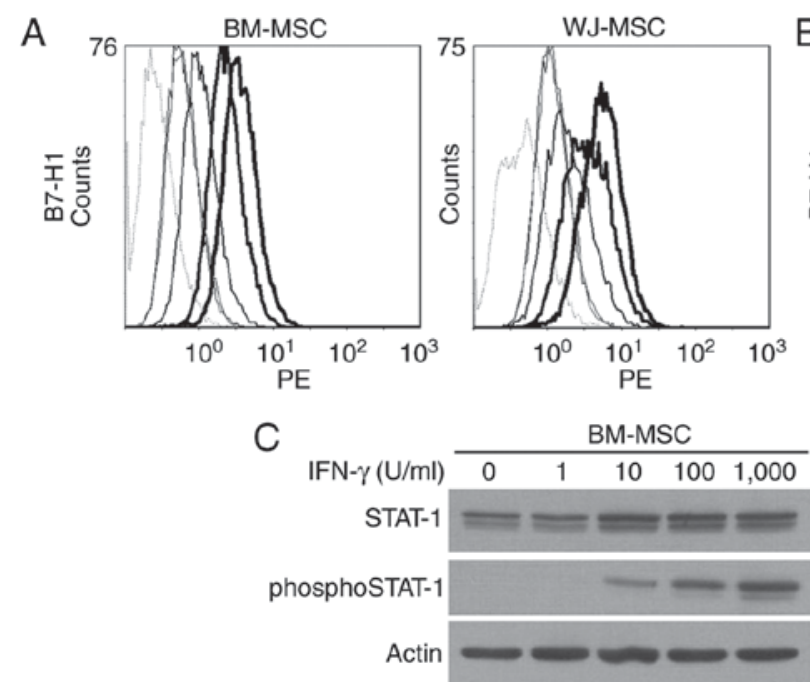
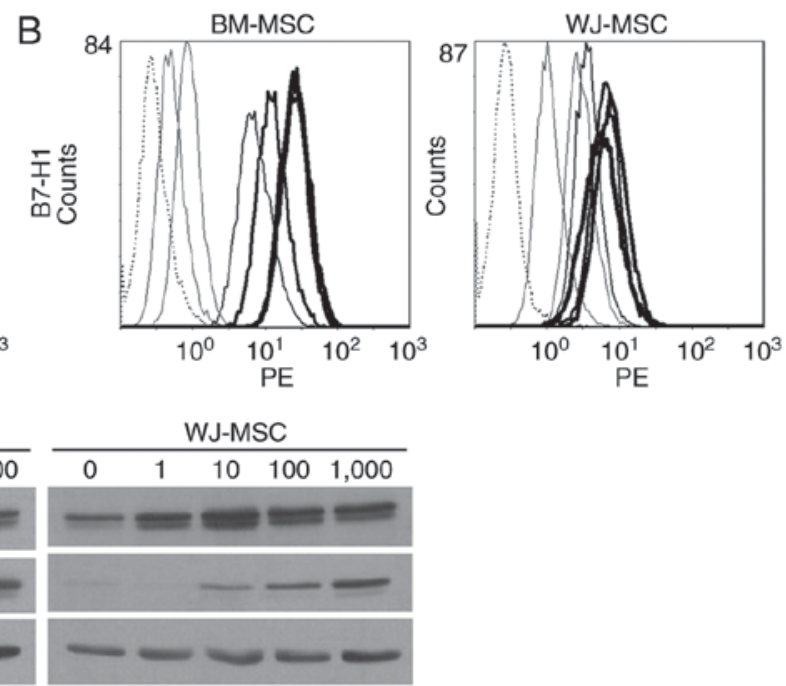

Figure 2. Dose- and time-dependent effects of IFN- $\gamma$ on B7-H1 and STAT-1 expression in MSCs. (A) BM and WJ-MSCs were stimulated with different doses of IFN- $\gamma$ for $24 \mathrm{~h}$. B7-H1 expression was analyzed by flow cytometry. Expression increased in a dose-dependent manner (dotted line, IgG control; line thickness increases according to dose: 0,1,10, 100 and 1,000 U/ml). (B) Time course of B7-H1 expression induced by 1,000 U/ml of IFN- $\gamma$. Expression was increased to a maximum $48 \mathrm{~h}$ following IFN- $\gamma$ treatment (dotted line, IgG control; line thickness increases according to the time: $0,6,12,24,48$ and $72 \mathrm{~h}$ of treatment). (C) MSCs were incubated with different doses IFN- $\gamma$ for $24 \mathrm{~h}$. Immunoblot analysis of STAT-1, phospho-STAT-1, and actin expression in MSCs. Phospho-STAT-1 was elevated in a dose-dependent manner in BM- and WJ-MSCs. IFN, interferon; STAT, signal transducer and activator of transcription; PE, phycoerythrin-conjugated antibodies; BM, bone marrow; WJ, Wharton's jelly; MSCs, mesenchymal stem cells; B7-H1, B7 homolog 1. 
A

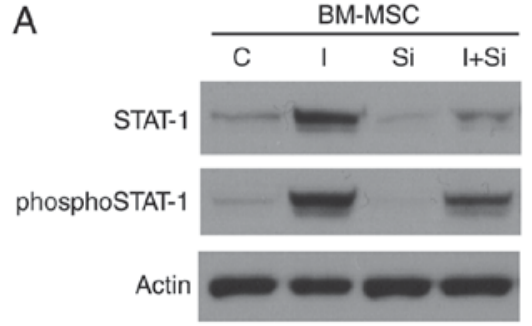

B

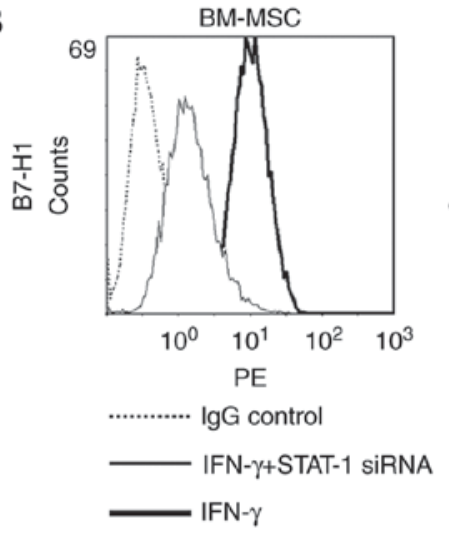

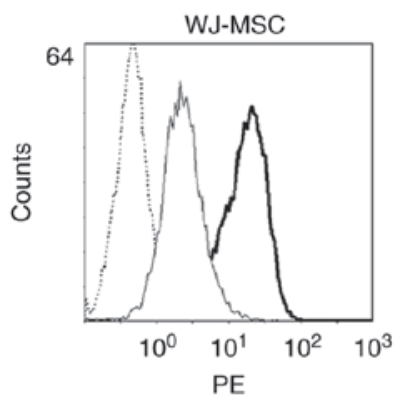
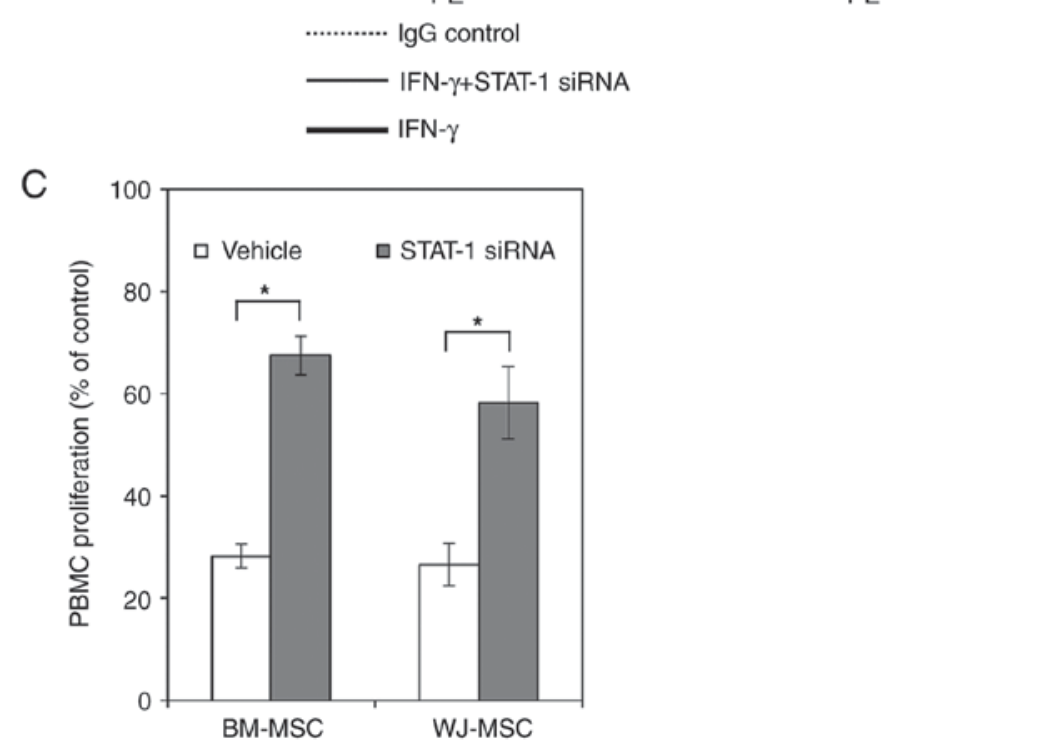

Figure 3. IFN- $\gamma$-induced STAT-1 pathway and B7-H1 expression in MSCs. (A) To downregulate STAT-1 activity, MSCs were transfected with siRNA targeting STAT-1. The expression levels of phospho-STAT-1, STAT-1, and actin in these transfected MSCs were detected by immunoblotting. (B) Downregulation of STAT-1 activity effectively induced a decrease in B7-H1 expression analyzed by flow cytometry in IFN- $\gamma$-treated MSCs. (C) T cell proliferation in STAT-1 siRNA treated MSCs was significantly restored compared to no siRNA treatment ("P $<0.05$; error bars represent standard deviation). The proliferation of $\mathrm{T}$ cells stimulated with phytohemagglutinin-induced PBMCs alone was used as a control. Data measured were relative to this control. si, small interfering RNA to STAT-1; I, IFN- $\gamma$ treatment; IFN, interferon; STAT, signal transducer and activator of transcription; si, small interfering; PE, phycoerythrin-conjugated antibodies; BM, bone marrow; WJ, Wharton's jelly; MSCs, mesenchymal stem cells; B7-H1, B7 homolog 1; PBMCs, peripheral blood mononuclear cells; IgG, immunoglobin.

next assessed. To inhibit PI3K/Akt activity, combined treatment with the PI3K inhibitor LY294002 and IFN- $\gamma$ was used (Fig. 4A). However, the expression of B7-H1 did not alter when LY294002 and IFN- $\gamma$ were administered together (Fig. 4B and C).

\section{Discussion}

Properties of MSCs include strong immune modulating effects and immunosuppressive functions, resulting in a number of researchers hypothesizing that they may be used for cell therapy (16). Although numerous studies have documented the immunosuppressive activities of MSCs and their potential therapeutic use in animals and humans, the underlying mechanisms are only partially known. A number of studies on the immunosuppressive activities of MSCs have focused on paracrine mechanisms, via soluble factors including IDO, hepatocyte growth factor, transforming growth factor- $\beta$, interleukin-10, prostaglandin E2, and unidentified factors (10). Cell-to-cell contact-associated mechanisms have been implicated in these effects, via B7-H1 and Fas ligand. MSCs are not immunosuppressive alone, and their immunosuppressive ability is greatly increased under inflammatory conditions in which pro-inflammatory cytokines are present. Therefore, MSCs require 'licensing' by pro-inflammatory cytokines including INF- $\gamma$ (16). These licensed MSCs inhibit the immune function of $\mathrm{T}$ cells through cell-to-cell direct contact and through the production of soluble factors including IDO (17).

IDO induces immunosuppression of T cells by promoting the catabolism of tryptophan and by stimulating tryptophan metabolites to increase cell cycle arrest and apoptosis in these cells (7). It has been demonstrated that IFN- $\gamma$-induced IDO production in MSCs not only results from sequential activation of Jak and STAT-1 proteins, however also involves IFN- $\gamma$-induced activation of kinases including PI3K. These results indicate that these are the key signaling elements that regulate IFN- $\gamma$-induced IDO production, thereby regulating the immunosuppressive phenotype of MSCs; this suggests a potential strategy to improve immune regulation by MSCs during disease treatment (7).

The expression of B7-H1 is markedly induced by IFN- $\gamma(11)$. It was confirmed that the expression levels of STAT-1 and phospho-STAT-1 in MSCs were dose-dependently increased by IFN- $\gamma$ (Fig. 2). When STAT-1 siRNA was used, B7-H1 expression on IFN- $\gamma$-treated MSCs was decreased and 
A
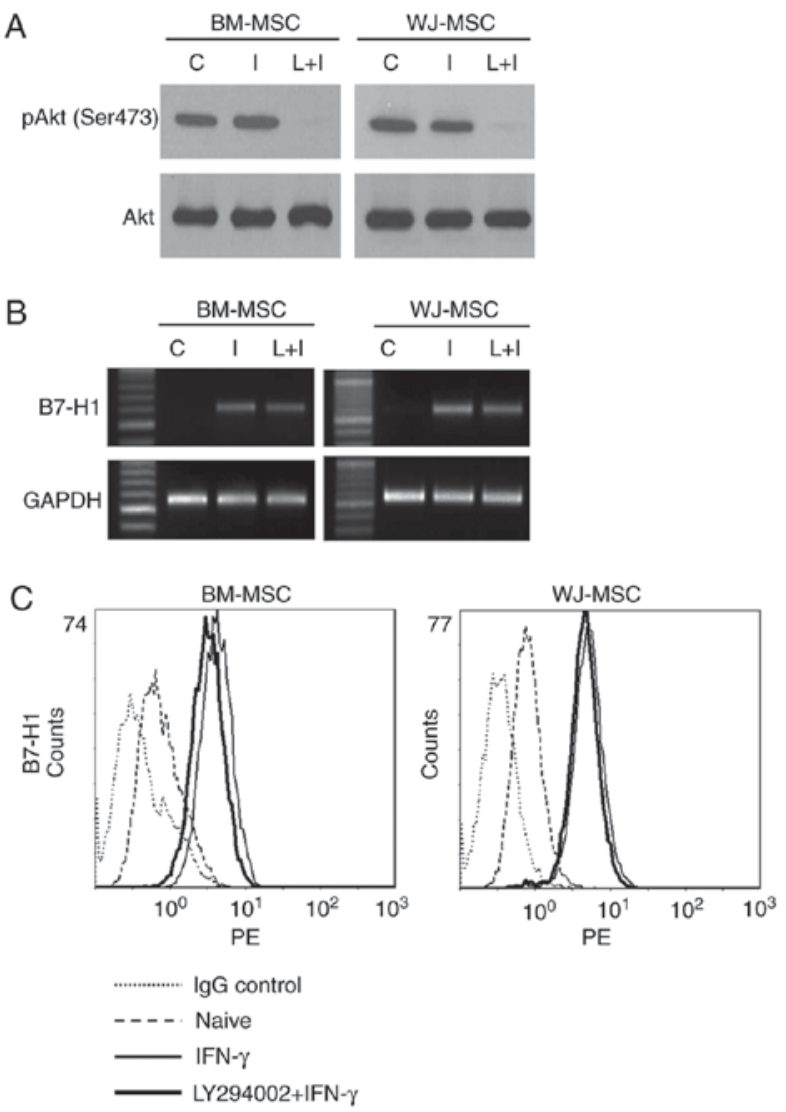

Figure 4. IFN- $\gamma$-mediated PI3K/Akt pathway and B7-H1 expression in MSCs. (A) To inhibit PI3K/Akt activity, MSCs were incubated with $10 \mu \mathrm{M}$ LY294002 (PI3K inhibitor) for $1 \mathrm{~h}$ and then treated with $1,000 \mathrm{U} / \mathrm{ml} \mathrm{IFN}-\gamma$ for $10 \mathrm{~min}$. The expression level of phospho-Akt and Akt in MSCs was detected by immunoblotting. LY294002 treatment blocked phospho-Akt expression; C, control; I, IFN- $\gamma$, L, LY294002 treatment. (B) Following treatment with IFN- $\gamma$ for $24 \mathrm{~h}$ in the presence or absence of LY294002, mRNA levels of B7-H1 in MSCs were determined by semi-quantitative reverse transcription polymerase chain reaction analysis, and (C) the expression B7-H1 was determined by flow cytometry. IFN, interferon; PI3K phosphatidylinositol-3-kinase; C, control; I, IFN- $\gamma$, L, LY294002 treatment; $\mathrm{PE}$, phycoerythrin-conjugated antibodies; BM, bone marrow; WJ, Wharton's jelly; MSCs, mesenchymal stem cells; B7-H1, B7 homolog 1; IgG, immunoglobin; p, phosphor; Akt, RAC- $\alpha$ serine/threonine-protein kinase.

T cell proliferation was restored (Fig. 3). However, LY294002, a PI3K signaling inhibitor, did not inhibit the activation of B7-H1 by IFN- $\gamma$ (Fig. 4).

The immunosuppression of T cells, mediated by increases in tryptophan metabolites via IFN- $\gamma$-induced IDO production in MSCs, is due to the action of and/or interaction with the Jak/STAT-1 pathway and the Akt/PI3K pathway (7). However, as demonstrated in the present study, B7-H1 expression in MSCs was mediated only by the Jak/STAT-1 pathway, and not by the Akt/PI3K pathway (Fig. 4). These results suggested that the mechanism of direct cell-cell contact-induced immunosuppression, mediated by interactions between B7-H1 molecules on MSCs and T cells, differed from that of soluble factors including IDO and cell-mediated signaling within MSCs.

B7-H1 is expressed not only in MSCs, however also in non-lymphoid tissue cells and certain tumor cells, and has been previously demonstrated to be induced by several cytokines during inflammatory conditions. In addition, anti-PD-L1 drugs are currently being studied for their use as anticancer drugs (18). Previously, it was reported that tumor-MSCs exhibit immunosuppressive effects on in vitro stimulated T lymphocytes, primarily through IDO, similar to those observed for BM-derived MSCs (18). Tumor-MSC numbers are directly proportional to the tumor volume and are inversely associated with the frequency of tumor-infiltrating leukocytes (19). This suggests that tumor-MSCs inhibit the antitumor response (20). It is thought that immunosuppression by tumor-MSCs may be due to the inhibitory effect of direct contact between cells, in addition to the effects of IDO (13). If the B7-H1-associated mechanism is verified for tumor-MSCs, it may be possible to use anti-Jak/STAT-1 pathway drugs to increase the antitumor effect. Previously, PD-L1 inhibitors and PD-1 inhibitors were established as anticancer drugs (21). Although they reduce the immunosuppressive effects of cancer cells on T cells, they are likely to inhibit similar MSC-associated effects on $\mathrm{T}$ cells expressing B7-H1, thereby enhancing anti-cancer responses (22). In particular, the emergence of STAT-1 inhibitors may be useful for enhancing $\mathrm{T}$ cell-mediated anti-cancer effects, by blocking both the inhibition of T7 cell proliferation and the immunosuppressive activities of soluble factors secreted by B7-H1-expressing MSCs.

Blocking the STAT-1 pathway increases T cell-mediated anti-tumor effects and graft-vs.-tumor effects by eliminating MSC-mediated T cell proliferation and mitotic inhibition (13). Clinical trials have been initiated for the treatment and prevention of severe acute graft-vs. host disease following allogeneic stem cell transplantation using MSCs $(16,23)$. STAT-1 inhibitors may be used when tumor recurrence is imminent, during the treatment of graft-vs. host disease with MSC cell therapy, as the STAT-1 pathway utilizes both IDO production and the direct contact effect based on B7-H1 cell-cell interactions (16). According to the results of the present study, the graft-vs. tumor effect may be temporarily augmented using STAT-1 inhibitors rather than Akt/PI3K inhibitors. Further animal tests and clinical trials are required to verify this.

The present study demonstrated that MSCs expressing IFN- $\gamma$-induced B7-H1 were induced by STAT-1 signaling, but not PI3K/Akt, to induce immunosuppressive effects on $\mathrm{T}$ cells. Understanding the mechanisms underlying the immunosuppressive effects on $\mathrm{T}$ cells induced by direct contact with B7-H1 in MSCs may aid in elucidating the properties of tumor-MSCs. The results of the present study may be applied to the induction of graft-vs.-host disease or graft-vs. tumor effects following allogeneic hematopoietic stem cell transplantation. The present study also provided insights into the application of cell therapy for $\mathrm{T}$ cell immunosuppression for the treatment of autoimmune diseases.

\section{Acknowledgements}

Not applicable.

\section{Funding}

The authors thank the Ajou University Medical Center for funding (grant no. M-2015-C0460-00110). 


\section{Availability of data and materials}

All data generated or analyzed during this study are included in this published article.

\section{Authors' contributions}

JP conceived the presented idea. JEP, IKJ and HJJ designed the research. JEP, IKJ, HJJ, DHL and KCL performed the research and analyzed the data. OKN designed the figures. JEP, IKJ and HJJ wrote the paper. JEP and IKJ edited the manuscript. All authors read and approved the final manuscript.

\section{Ethics approval and consent to participate}

The Institutional Review Board of Ajou University Hospital (Suwon, Korea) approved the present study (AJIRB-GN3-07-208), and all samples were obtained with informed consent.

\section{Consent for publication}

Not applicable.

\section{Competing interests}

The authors declare that they have no competing interests.

\section{References}

1. Friedenstein AJ, Chailakhyan RK and Gerasimov UV: Bone marrow osteogenic stem cells: In vitro cultivation and transplantation in diffusion chambers. Cell Tissue Kinet 20: 263-272, 1987.

2. Lv FJ, Tuan RS, Cheung KM and Leung VY: Concise review: The surface markers and identity of human mesenchymal stem cells. Stem Cells 32: 1408-1419, 2014.

3. Dominici M, Le Blanc K, Mueller I, Slaper-Cortenbach I, Marini F, Krause D, Deans R, Keating A, Prockop D and Horwitz E: Minimal criteria for defining multipotent mesenchymal stromal cells. The International Society for Cellular Therapy position statement. Cytotherapy 8: 315-317, 2006.

4. Grégoire C, Lechanteur C, Briquet A, Baudoux É, Baron F, Louis E and Beguin Y: Review article: Mesenchymal stromal cell therapy for inflammatory bowel diseases. Aliment Pharmacol Ther 45: 205-221, 2017.

5. Le Blanc K, Tammik C, Rosendahl K, Zetterberg E and Ringdén O: HLA expression and immunologic properties of differentiated and undifferentiated mesenchymal stem cells. Exp Hematol 31: 890-896, 2003.

6. Lee MW, Kim DS, Yoo KH, Kim HR, Jang IK, Lee JH, Kim SY, Son MH, Lee SH, Jung HL, et al: Human bone marrow-derived mesenchymal stem cell gene expression patterns vary with culture conditions. Blood Res 48: 107-114, 2013.

7. Mounayar M, Kefaloyianni E, Smith B, Solhjou Z, Maarouf OH, Azzi J, Chabtini L, Fiorina P, Kraus M, Briddell R, et al: PI3ko and STAT1 interplay regulates human mesenchymal stem cell immune polarization. Stem Cells 33: 1892-1901, 2015.
8. Wang G, Zhang S, Wang F, Li G, Zhang L and Luan X: Expression and biological function of programmed death ligands in human placenta mesenchymal stem cells. Cell Biol Int 37: 137-148, 2013.

9. Yan Z, Zhuansun Y, Liu G, Chen R, Li J and Ran P: Mesenchymal stem cells suppress $\mathrm{T}$ cells by inducing apoptosis and through PD-1/B7-H1 interactions. Immunol Lett 162: 248-255, 2014.

10. Tipnis S, Viswanathan C and Majumdar AS: Immunosuppressive properties of human umbilical cord-derived mesenchymal stem cells: Role of B7-H1 and IDO. Immunol Cell Biol 88: 795-806, 2010.

11. Jang IK, Yoon HH, Yang MS, Lee JE, Lee DH, Lee MW, Kim DS and Park JE: B7-H1 inhibits T cell proliferation through MHC class II in human mesenchymal stem cells. Transplant Proc 46: 1638-1641, 2014.

12. Croitoru-Lamoury J, Lamoury FM, Caristo M, Suzuki K, Walker D, Takikawa O, Taylor R and Brew BJ: Interferon- $\gamma$ regulates the proliferation and differentiation of mesenchymal stem cells via activation of indoleamine 2,3 dioxygenase (IDO). PLoS One 6: e14698, 2011.

13. Zaidi MR and Merlino G: The two faces of interferon- $\gamma$ in cancer. Clin Cancer Res 17: 6118-6124, 2011.

14. Lee MW, Choi J, Yang MS, Moon YJ, Park JS, Kim HC and Kim YJ: Mesenchymal stem cells from cryopreserved human umbilical cord blood. Biochem Biophys Res Commun 320: 273-278, 2004

15. Jang IK, Ahn JI, Shin JS, Kwon YS, Ryu YH, Lee JK, Park JK, Song KY, Yang EK and Kim JC: Transplantation of reconstructed corneal layer composed of corneal epithelium and fibroblasts on a lyophilized amniotic membrane to severely alkali-burned cornea. Artif Organs 30: 424-431, 2006.

16. Kim N, Im KI, Lim JY, Jeon EJ, Nam YS, Kim EJ and Cho SG: Mesenchymal stem cells for the treatment and prevention of graft-versus-host disease: Experiments and practice. Ann Hematol 92: 1295-1308, 2013.

17. Chinnadurai R, Copland IB, Patel SR and Galipeau J: IDO-independent suppression of $\mathrm{T}$ cell effector function by IFN- $\gamma$-licensed human mesenchymal stromal cells. J Immunol 192: 1491-1501, 2014.

18. Ritprajak P and Azuma M: Intrinsic and extrinsic control of expression of the immunoregulatory molecule PD-L1 in epithelial cells and squamous cell carcinoma. Oral Oncol 51: 221-228, 2015.

19. Liotta F, Querci V, Mannelli G, Santarlasci V, Maggi L, Capone M, Rossi MC, Mazzoni A, Cosmi L, Romagnani S, et al: Mesenchymal stem cells are enriched in head neck squamous cell carcinoma, correlates with tumour size and inhibit T-cell proliferation. Br J Cancer 112: 745-754, 2015.

20. Shou P, Chen Q, Jiang J, Xu C, Zhang J, Zheng C, Jiang M, Velletri T, Cao W, Huang Y, et al: Type I interferons exert anti-tumor effect via reversing immunosuppression mediated by mesenchymal stromal cells. Oncogene 35: 5953-5962, 2016.

21. Carbotti G, Barisione G, Airoldi I, Mezzanzanica D, Bagnoli M, Ferrero S, Petretto A, Fabbi M and Ferrini S: IL-27 induces the expression of IDO and PD-L1 in human cancer cells. Oncotarget 6: 43267-43280, 2015.

22. Seo SK, Seo DI, Park WS, Jung WK, Lee DS, Park SG, Choi JS, Kang MS, Choi YH, Choi I, et al: Attenuation of IFN- $\gamma$-induced B7-H1 expression by 15-deoxy-delta(12,14)-prostaglandin J2 via downregulation of the Jak/STAT/IRF-1 signaling pathway. Life Sci 112: 82-89, 2014

23. Burand AJ, Gramlich OW, Brown AJ and Ankrum JA: Function of cryopreserved mesenchymal stromal cells with and without interferon- $\gamma$ prelicensing is context dependent. Stem Cells 35: 1437-1439, 2017. 